\title{
ON THE DEGREE OF APPROXIMATION OF A CLASS OF FUNCTIONS BY MEANS OF FOURIER SERIES
}

\section{S. M. MAZHAR}

ABSTRACT. In this paper degree of approximation of Lebesgue integrable functions by means of Fourier series is examined.

1. Let $f$ be a periodic function with period $2 \pi$ and integrable in the sense of Lebesgue. Let

$$
f(x) \sim \frac{a_{0}}{2}+\sum_{1}^{\infty}\left(a_{k} \cos k x+b_{k} \sin k x\right) .
$$

We write

$$
\phi(t)=\frac{1}{2}\{f(x+t)+f(x-t)-2 f(x)\} \quad \text { and } \quad \Phi(t)=\int_{0}^{t}|\phi(u)| d u .
$$

Let

$$
w_{1}(\delta)=w_{1}[f, x](\delta)=\sup _{|h| \leqslant \delta}\left\{\frac{1}{2 h} \int_{-h}^{h}|f(x+u)-f(x)| d u\right\} .
$$

It is clear that for $f \in C^{*}[0,2 \pi], w_{1}(\delta) \leqslant w[f](\delta)$, where $w[f](\delta)$ denotes the modulus of continuity of $f$.

Let $\Lambda=\left(\lambda_{n, k}\right), k=0,1,2, \ldots, n$, be a triangular matrix and let

$$
\sigma_{n}=\sum_{k=0}^{n} \lambda_{n, k} s_{k}
$$

where $\left\{s_{k}\right\}$ is a given sequence of numbers. $\sigma_{n}$ is called $n$th $\Lambda$-means of $\left\{s_{n}\right\}$. If $\sigma_{n} \rightarrow s$ as $n \rightarrow \infty$, we say that $\left\{s_{n}\right\}$ is summable $(\Lambda)$ to $s$. We suppose that $\left\{\lambda_{n, k}\right\}$ is nonnegative with $\sum_{k=0}^{n} \lambda_{n, k}=1, n=0,1, \ldots$ Then a necessary and sufficient condition for regularity of the $\Lambda$-method is $\lim _{n \rightarrow \infty} \lambda_{n, k}=0$ for each $k$.

For

$$
\lambda_{n, k}=\frac{p_{n-k}}{P_{n}}, \quad P_{n}=p_{0}+p_{1}+\cdots+p_{n}, p_{0}>0,
$$

the $\Lambda$-method reduces to the $\left(N, p_{n}\right)$ method. Similarly for $\lambda_{n, k}=p_{k} / P_{n}$, we get $\left(\bar{N}, p_{n}\right)$ means.

Received by the editors August 4, 1982.

1980 Mathematics Subject Classification. Primary 42A10; Secondary 42A24, 40 C05.

Key words and phrases. Degree of approximation, $\Lambda$-means, modulus of continuity.

${ }^{1}$ This research was supported by Kuwait University Research Council Grant no. SM 007. 
In what follows we assume that $C$ is a positive constant not necessarily the same at each occurrence.

2. In this paper we establish the following

THEOREM. Suppose for fixed $x, w_{1}(\delta)<\infty$ for $\delta \in(0, \pi]$, and let $\sigma_{n}(x)$ denote the nth $\Lambda$-means of the Fourier series of $f(x)$. If $\left\{\lambda_{n, k}\right\}$ is nondecreasing with respect to $k$, then

$$
\left|\sigma_{n}(x)-f(x)\right| \leqslant C \sum_{k=0}^{n} \frac{w_{1}(\pi /(k+1))}{k+1} \sum_{\nu=0}^{k} \lambda_{n, n-\nu} .
$$

3. Proof. We have

$$
\begin{aligned}
\sigma_{n}(x)-f(x) & =\frac{2}{\pi} \int_{0}^{\pi} \phi(t) \sum_{k=0}^{n} \lambda_{n, k} D_{k}(t) d t \\
& =\frac{2}{\pi}\left(\int_{0}^{\pi / n+1}+\int_{\pi / n+1}^{\pi}\right)=I_{1}+I_{2}, \text { say, }
\end{aligned}
$$

where

$$
D_{k}(t)=\frac{\sin \left(k+\frac{1}{2}\right) t}{2 \sin t / 2}
$$

Since

$$
\Phi(t)=\int_{0}^{t}|\phi(u)| d u \leqslant \frac{1}{2} \int_{t}^{t}|f(x+u)-f(x)| d u \leqslant t w_{1}(t),
$$

it follows that

$$
\begin{aligned}
\left|I_{1}\right| & \leqslant \frac{2}{\pi} \int_{0}^{\pi / n+1}|\phi(t)| \sum_{k=0}^{n} \lambda_{n, k}\left(k+\frac{1}{2}\right) d t \leqslant \frac{2(n+1)}{\pi} \int_{0}^{\pi / n+1}|\phi(t)| d t \\
& \leqslant 2 w_{1}\left(\frac{\pi}{n+1}\right) \leqslant 2 \sum_{k=0}^{n} \frac{w_{1}(\pi /(k+1))}{k+1} \sum_{\nu=0}^{k} \lambda_{n, n-\nu}
\end{aligned}
$$

Let $\gamma_{n}$ be a sequence of linear functions on $[k, k+1]$ such that $\gamma_{n}(k)=\lambda_{n, n-k}$, $k=0,1,2, \ldots, n$, and let $F_{n}(t)=\int_{0}^{t} \gamma_{n}(u) d u, t \geqslant 0$. Then

$$
\begin{aligned}
F_{n}(k) & =\sum_{\nu=0}^{k-1} \frac{\gamma_{n}(\nu+1)+\gamma_{n}(\nu)}{2}=\sum_{\nu=0}^{k-1} \frac{\lambda_{n, n-\nu-1}+\lambda_{n, n-\nu}}{2} \\
& \leqslant \sum_{\nu=0}^{k} \lambda_{n, n-\nu} \leqslant 2 F_{n}(k) .
\end{aligned}
$$

Using the well-known estimate of McFadden [5],

$$
\left|\sum_{k=a}^{b} \lambda_{n, n-k} e^{i(n-k) t}\right| \leqslant 2(2 \pi+1) F_{n}\left(\frac{\pi}{t}\right),
$$


where $0 \leqslant a \leqslant b \leqslant \infty, 0<t \leqslant \pi$ and $n$ is any nonnegative integer, we have

$$
\begin{aligned}
\left|I_{2}\right| \leqslant & \frac{2}{\pi} \int_{\pi / n+1}^{\pi}|\phi(t)|\left|\sum_{k=0}^{n} \lambda_{n, k} D_{k}(t)\right| d t \leqslant C \int_{\pi / n+1}^{\pi}\left|\frac{\phi(t)}{t}\right| F_{n}\left(\frac{\pi}{t}\right) d t \\
= & C\left\{\left[\frac{\Phi(t)}{t} F_{n}\left(\frac{\pi}{t}\right)\right]_{\pi / n+1}^{\pi}+\int_{\pi / n+1}^{\pi} \frac{\Phi(t)}{t^{2}} F_{n}\left(\frac{\pi}{t}\right) d t\right. \\
& \left.+\int_{\pi / n+1}^{\pi} \frac{\Phi(t)}{t} F_{n}^{\prime}\left(\frac{\pi}{t}\right) \cdot \frac{\pi}{t^{2}} d t\right\} \\
= & C\left\{\frac{\Phi(\pi)}{\pi} F_{n}(1)-\frac{(n+1)}{\pi} \Phi\left(\frac{\pi}{n+1}\right) F_{n}(n+1)\right. \\
& \leqslant C w_{1}(\pi) \lambda_{n, n}+C \sum_{k=1}^{n} \int_{k}^{k+1} \frac{\pi}{t} w_{1}\left(\frac{\pi}{t}\right) F_{n}(t) d t+C \sum_{k=1}^{n+1} \int_{k}^{k+1} w_{1}\left(\frac{\pi}{t}\right) \gamma_{n}(t) d t \\
& =I_{21}+I_{22}+I_{23}, \quad \text { say. }
\end{aligned}
$$

Obviously,

$$
I_{21} \leqslant C \sum_{k=0}^{n} w_{1}\left(\frac{\pi}{k+1}\right) \lambda_{n, n-k} \leqslant C \sum_{k=0}^{n} \frac{w_{1}(\pi /(k+1))}{k+1} \sum_{\nu=0}^{k} \lambda_{n, n-\nu}
$$

and

$$
\begin{aligned}
I_{22} & \leqslant C \sum_{k=1}^{n} \frac{w_{1}(\pi / k)}{k} F_{n}(k+1) \leqslant C \sum_{k=0}^{n-1} \frac{w_{1}(\pi /(k+1))}{k+1} \sum_{\nu=0}^{k} \lambda_{n, n-\nu} \\
& \leqslant C \sum_{k=0}^{n} \frac{w_{1}(\pi /(k+1))}{k+1} \sum_{\nu=0}^{k} \lambda_{n, n-\nu} .
\end{aligned}
$$

Similarly,

$$
\begin{aligned}
I_{23} & \leqslant C \sum_{k=1}^{n} w_{1}\left(\frac{\pi}{k}\right)\left(\frac{\gamma_{n}(k)+\gamma_{n}(k+1)}{2}\right) \leqslant C \sum_{k=1}^{n} w_{1}\left(\frac{\pi}{k}\right) \lambda_{n, n-k} \\
& \leqslant C \sum_{k=0}^{n} w_{1}\left(\frac{\pi}{k+1}\right) \lambda_{n, n-k} \leqslant C \sum_{k=0}^{n} \frac{w_{1}(\pi /(k+1))}{k+1} \sum_{\nu=0}^{k} \lambda_{n, n-\nu} .
\end{aligned}
$$

Thus

$$
\left|I_{2}\right| \leqslant C \sum_{k=0}^{n} \frac{w_{1}(\pi /(k+1))}{k+1} \sum_{\nu=0}^{k} \lambda_{n, n-\nu} .
$$

From (3.1) and (3.2) the proof of our theorem follows.

4. Taking $\lambda_{n, k}=p_{n-k} / P_{n}$, where $\left\{p_{k}\right\}$ is a positive nonincreasing sequence, we deduce from (2.1) the following theorem due to Markiewicz [4]; the case $p_{n}=1$ is due to Aljančić, Bojanic and Tomić [1]. 
COROllary 1. If for fixed $x, w_{1}(\delta)<\infty$ for $\delta \in(0, \pi]$, then

$$
\left|t_{n}(x)-f(x)\right| \leqslant \frac{C}{P_{n}} \sum_{k=0}^{n} \frac{P_{k}}{k+1} w_{1}\left(\frac{\pi}{k+1}\right),
$$

where $t_{n}(x)$ denotes the $\left(N, p_{n}\right)$ means of the Fourier series of $f(x)$.

This result in weaker form, where $w_{1}$ is replaced by $w$, is due to Holland, Sahney and Tzimbalario [3]. For related results concerning Cesàro summability see Obrechkoff [6] and Flett [2].

COROLlaRY 2. If $\left\{\lambda_{n, k}\right\}$ is a nonincreasing sequence with respect to $k$, then under the hypothesis of the theorem;

$$
\left|\sigma_{n}(x)-f(x)\right| \leqslant C \sum_{k=0}^{n} \lambda_{n, k} w_{1}\left(\frac{\pi}{k+1}\right) .
$$

Proof. Let $t_{n}^{*}(x)$ denote the $(C, 1)$ mean of the Fourier series. Then taking $\lambda_{n, k}=(n+1)^{-1}$ in our Theorem, we have

$$
\left|t_{n}^{*}(x)-f(x)\right| \leqslant \frac{C}{n+1} \sum_{k=0}^{n} w_{1}\left(\frac{\pi}{k+1}\right) .
$$

Using a partial summation formula of Abel,

$$
\begin{aligned}
\sigma_{n}(x)-f(x) & =\sum_{\nu=0}^{n} \lambda_{n, \nu}\left(s_{\nu}(x)-f(x)\right) \\
& =\sum_{\nu=0}^{n-1} \Delta \lambda_{n, \nu}(\nu+1)\left(t_{\nu}^{*}(x)-f(x)\right)+\lambda_{n, n}(n+1)\left(t_{n}^{*}(x)-f(x)\right) \\
& =\sum_{\nu=0}^{n}(\nu+1)\left(t_{\nu}^{*}(x)-f(x)\right) \Delta \lambda_{n, \nu} .
\end{aligned}
$$

Since $\Delta \lambda_{n, \nu} \geqslant 0$ we have, from (4.1),

$$
\begin{aligned}
\left|\sigma_{n}(x)-f(x)\right| & \leqslant C \sum_{\nu=0}^{n} \Delta \lambda_{n, \nu} \sum_{k=0}^{\nu} w_{1}\left(\frac{\pi}{k+1}\right) \\
& =C \sum_{k=0}^{n} w_{1}\left(\frac{\pi}{k+1}\right) \sum_{\nu=k}^{n} \Delta \lambda_{n, \nu}=C \sum_{k=0}^{n} \lambda_{n, k} w_{1}\left(\frac{\pi}{k+1}\right) .
\end{aligned}
$$

The author thanks the referee for suggesting the proof of Corollary 2 and also for improvement in the presentation of this paper.

\section{REFERENCES}

1. S. Aljančić, R. Bojanic and M. Tomić, On the degree of convergence of Fejer-Lebesgue sums, Enseign. Math. (2) 15 (1969), 21-28.

2.T. M. Flett, On the degree of approximation to a function by the Cesàro means of a Fourier series, Quart. J. Math. Oxford Ser. (2) 7 (1956), 81-95.

3. A. S. B. Holland, B. N. Sahney and J. Tzimbalario, On the degree of approximation of a class of functions by means of Fourier series, Acta. Sci. Math. (Szeged) 38 (1976), 69-72.

4. T. Markiewicz, Sur l'approximation des fonctions par les moyennes de Nörlund des séries de Fourier et leurs conjugées, Funct. Approx. Comment. Math. 8 (1980), 77-83.

5. L. McFadden, Absolute Nörlund summability, Duke Math. J. 9 (1942), 168-207.

6. N. Obrechkoff, Sur la sommation des séries trigonométriques de Fourier par les moyennes arithmétiques, Bull. Soc. Math. France 62 (1934), 84-109.

Department of Mathematics, KuWait University, Kuwait 